\title{
La disrupción escolar: un buen pretexto para las reflexiones docentes
}

\author{
School Disruption: A Good Excuse for Teacher Reflections
}

\author{
Salomón Vásquez Villanueva ${ }^{1}$, Eloy Colque ${ }^{2}$ y Wilma Villanueva ${ }^{3}$ \\ Universidad Peruana Unión, Lima-Perú ${ }^{123}$ \\ Orcid ID: https://orcid.org/0000-0001-8824-6176 ${ }^{1}$ \\ Orcid ID: https://orcid.org/0000-0003-4555-0674² \\ Orcid ID: https://orcid.org/0000-0001-8138-8235
}

Recibido: 30 de octubre de 2018

Aceptado: 04 de julio 2019

\begin{abstract}
Resumen
El objetivo de este trabajo es catalogar los factores predictores, las consecuencias y las soluciones de la disrupción escolar, en el contexto de la convivencia escolar y el aprendizaje de los alumnos. Para lograr este objetivo, se han abordado diversas investigaciones sobre la disrupción escolar: sus concepciones, factores predictores, conductas disruptivas, consecuencias, soluciones y teorías. En conclusión, la prevención y la solución de las conductas disruptivas son muy importantes. En este sentido, se debe realizar un análisis profundo y reflexivo sobre los contextos, los factores predictores y las consecuencias de dichas conductas; además interpretar acertadamente estas, creando un ambiente de comunicación y convivencia, de acuerdo las teorías y los enfoques sistémicos.
\end{abstract}

Palabras clave: disrupción escolar, factores predictores, consecuencias, soluciones.

\begin{abstract}
The aim of this research is to catalog the predictive factors, consequences and solutions of school disruption, in the context of school coexistence and student learning. To achieve this goal, several investigations on school disruption have been addressed concerning to their conceptions, their predictive factors, disruptive behaviors, consequences, solutions and theories. In conclusion, the prevention and solution of disruptive behaviors are very important. In this sense, a deep and reflective analysis must be carried out on the contexts, the predictive factors and the consequences; also interpret disruptive behaviors correctly,
\end{abstract}

${ }^{1}$ Correspondencia al autor

E-mail: salomonv@upeu.edu.pe. 
creating an environment of communication and coexistence, according to theories and systemic approaches.

Keywords: school disruption, predictive factors, consequences, solutions.

\section{Introducción}

Al situar el pensamiento reflexivo y crítico de los docentes, incluso de los investigadores en el espacio escolar, nace de inmediato la percepción sobre la convivencia escolar, en la cual se describen conductas, comportamientos y actitudes de los profesores, alumnos, padres de familia y directivos. Las relaciones y las interrelaciones de los mismos se manifiestan en forma positiva y negativa; esta última genera los diversos conflictos educativos relacionados con "la indisciplina, la disrupción y en menor medida la desidia docente” (Penalva López, 2018, p. 43).

Los problemas diversos y los conflictos escolares se incrementan con el devenir de los años. En este contexto, la disrupción escolar es un tema de interés, de gran preocupación y de mucha importancia para los docentes, los padres de familia y los investigadores, quienes deben entenderla con mucha precisión lo que significa, cuáles son sus factores predictores, cuáles son sus consecuencias, cómo prevenirla o evitarla, conociendo de esta manera su ligazón con otros conflictos; los cuales atentan contra los procesos de aprendizaje de los alumnos, el clima y la convivencia escolar. En este sentido, el objetivo del presente estudio es catalogar los factores predictores y las consecuencias de la disrupción, observados durante la convivencia escolar y el aprendizaje.

\section{Términos relacionados con la disrupción escolar}

En el ámbito de la escuela, con el propósito de conocer y analizar los problemas y los conflictos diversos que atentan contra la convivencia y el clima escolar, de inmediato se circunscriben algunos conceptos, cuya relación semántica se debe dilucidar. Entre 
otros, estos conceptos son: agresión escolar, bullying, convivencia escolar, hostigamiento escolar y violencia; todos ellos explicados y analizados en el contexto de "las relaciones sociales en las escuelas".

La agresión escolar es entendida en los términos de insultos lingüísticos (con palabras) destinados a los compañeros y familiares (Rosário et al., 2012). El bullying es el hostigamiento ente iguales (Moreno, 2007; Chaux, 2012; en Beltrán, Torrado, y Vargas, 2016). La convivencia escolar es "una construcción personal y social", la cual se orienta hacia "la creación de un mundo común", cuya convivencia (de docentes, estudiantes, padres de familia y directivos) se fortalece mediante la práctica de los valores: equidad, justicia, aceptación, respeto, confianza y pluralismo; inclusive es considerada es un proceso de interrelación personal y colectiva (Pérez, 2001; Maturana, 2002; Mockus, 2002; Fierro, 2013; en Díaz Better and Sime Poma, 2016).

El hostigamiento escolar viene a ser "una forma de violencia entre iguales", no se da entre desiguales. Es el maltrato que se da en forma reiterada, prolongada y de manera intencionada, cuya agresión puede ser física y/o psicológica, debido al desequilibrio de la interrelación por el poder y/o la fuerza, en un escenario donde se encuentran: agresor, víctima y espectador o testigo (Olweus, 1993, en Beltrán, Torrado, and Vargas, 2016).

La violencia escolar presenta los siguientes episodios: "1. Disrupción en las aulas, 2. Actitudes violentas entre alumnos y profesores, 3. Maltrato entre compañeros (bullying), 4. Vandalismo y daños materiales, 5. Violencia física (como forma de convivencia), 6. Violencia emocional (como forma de convivencia), 7. Violencia verbal (como forma de convivencia), 8. Abuso o acoso sexual” (Luciano and Marín, 2011). Asimismo, comprende un conjunto de actos; por ejemplo, hostigamiento, agresión, amenazas, daño; expresados intencional y sistemáticamente, cuyas formas y niveles son diversos: disrupción, problemas de disciplina, bullying (hostigamiento entre iguales), 
vandalismo y daños materiales, violencia física y acoso sexual (Moreno, 2007; Chaux, 2012; en Beltrán et al., 2016). Igualmente es un constructo multifactorial, "un proceso de interacción” más que "un fenómeno individual” (Domínguez Alonso, Álvarez Roales, and Vázquez Varela, 2017).

\section{La disrupción: aproximaciones conceptuales}

Recibe este nombre el "comportamiento del alumno o del grupo que busca romper el proceso de enseñanza-aprendizaje" (Uruñuela Nájera, 2007, p. 103). Es parte de los conflictos. Para Capllonch Bujosa, Figueras Comas, and Lleixà Arribas (2014, citando a Torrego, 2006 y Vizcarra, Macazaga, Perera, Maiztegui, Arostegui y Gasituaga, 2007), entre "los conflictos más comunes en los centros escolares" se "distingue los siguientes: violencia psicológica, física y estructural, disrupción en las aulas, vandalismo, procesos de disciplina, bullying, acoso sexual, absentismo y deserción escolar, así como, problemas de seguridad en el centro escolar".

Las escuelas no solamente son espacios establecidos, organizados y sistematizados para la enseñanza y el aprendizaje, también han sido destinados para la convivencia, la cual es compleja; además deja la evidencia de las conductas disruptivas que deben ser interpretadas convenientemente, con urgencia y a tiempo. Por ello, que la disrupción es el "comportamiento del alumno o del grupo que busca romper el proceso de enseñanzaaprendizaje, que implícita o explícitamente se plantea que este proceso no llegue a establecerse" (Álvarez Hernández, Castro Pañeda, González-González de Mesa, Álvarez Martino, and Campo Mon, 2016).

Durante los últimos años, los estudios revelan que el escenario de la escuela presenta muchas dificultades durante el desarrollo del proceso enseñanza-aprendizaje. Es objetiva la presencia de conductas y comportamientos negativos en el aula, es decir, la disrupción, cuyas causas y soluciones requieren estudios y análisis (Álvarez Hernández, 
Castro Pañeda, González-González de Mesa, Álvarez Martino, and Campo Mon, 2016). La disrupción siempre ocurre dentro del aula, constituye "comportamientos con los que el alumnado dificulta al profesorado impartir clase y al resto de compañeros interesados en seguirla con aprovechamiento" (Domínguez Alonso et al., 2017, p. 339). Para Tapia Sisalema and Rodríguez Baque (2018), la disrupción es "una actitud negativa", "un desacato de órdenes". De acuerdo con los autores consultados, la disrupción tiene el sentido de comportamientos, conductas, conflictos y actitudes personales y grupales, en el espacio denominado aula en el centro educativo o institución educativa.

\section{Factores predictores de la disrupción}

En este espacio, se presentan algunos factores predictores: las conductas de los docentes, las conductas de los padres de familia, el fenómeno migratorio y otros.

\section{Las conductas de los docentes, factor predictor determinante}

Aunque es muy difícil separarlos o desligarlos de los demás actores, los docentes y sus estilos de enseñanza, su actuación y convivencia favorecen la disrupción, y así se constituyen en un "fenómeno íntimamente ligado con el fracaso escolar"; tienen parte los factores involucrados en el proceso enseñanza-aprendizaje, incluso se suman los problemas del centro educativo. La indisciplina y la disrupción son conductas "fácil atribuirlas a factores externos, a la edad, a la capacidad de los alumnos, a las diferencias psicológicas, a las diferencias socio-económicas o culturales; todos estos factores están presentes e influyen en la mayor o menor frecuencia de estas conductas" (Uruñuela Nájera, 2007, p. 107).

La disrupción se desarrolla en el aula, en el ambiente de convivencia de los alumnos con sus pares y con el profesor; este es un factor determinante y predictivo de la ausencia o presencia de la disrupción, muchas veces se observa que "la acción educativa deja en manos de la improvisación decisiones que merecen ser bien pensadas, planificadas y 
evaluadas" (Ibarrola-García, 2014). El profesor-amparado por sus creencias, sus conocimientos y sus metodologías - tiene las condiciones para realizar la gestión del clima escolar, evitando los problemas relacionados con la convivencia escolar: disrupción, conflictos interpersonales, desmotivación, bullying, entre otros (Informe TALIS, 2009, en Ibarrola-García, 2014).

Definitivamente, "el profesorado actúa de forma muy diferente en sus clases en razón de su pensamiento pedagógico, ideología sociocultural y compromiso profesional" (Fernández-Larragueta, Fernández-Sierra, and Rodorigo, 2017, p. 492). Los factores que afectan la convivencia escolar son de índole social, impidiendo el desarrollo de habilidades sociales para manejar los conflictos sociales en el aula. También se suman los factores denominados relaciones interpersonales. Se necesita inteligencia emocional (Tapia Sisalema and Rodríguez Baque, 2018). Por otro lado, el género del docente es un predictor importante de los comportamientos disruptivos (Baños, Ortiz-Camacho, BaenaExtremera, and Zamarripa, 2018).

\section{Las conductas de los padres de familia, factor predictor poderoso}

La autoestima, la familia y la escuela dejan sus implicancias sobre el estatus sociométrico y la violencia escolar (Martínez Ferrer, Musitu Ochoa, Amador Muñoz, y Monreal Gimeno, 2012). Picazo-Zappino (2014) ha encontrado durante su investigación que "la disrupción familiar o los antecedentes psiquiátricos no serían relevantes"; sin embargo, operarían "de forma impulsiva y sin premeditación, motivado por un acontecimiento vital estresante, y con la finalidad de modificar el entorno" (p. 129). Además, los estilos de crianza parental tienen sus influjos sobre las conductas disruptivas de los niños (Armijos Piedra and Castro Ponce, 2018).

\section{El fenómeno migratorio: un factor predictor singular}


El fenómeno migratorio es un factor predictor muy singular. Aplicable para otros país y latitudes, Jacobo-Suárez ( 2017), en México, realiza un estudio sobre el fenómeno migratorio de los mexicanos a los Estados Unidos de America (EUA), cuyo retorno de cónyuges e hijos comprende la deportación (orden judicial de expulsión), la repatriación (retorno irregular después de dos años), retorno forzado ("firma la deportación voluntaria ante un juez") y el retorno voluntario (regreso voluntario "por circunstancias familiares o económicas”). Según estudio, 3.3 millones niños, quienes han nacido en EUA, tienen el padre o la madre con la disposición de deportación, por permanecer indocumentado, cuya deportación, además, le genera al niño la disrupción en la escuela. "Esta compleja realidad explica por qué cuando un adulto es deportado, especialmente si es el proveedor del hogar, es probable que su cónyuge e hijos le sigan en su regreso a México” (p. 2).

\section{Otros factores predictores}

Buitrago Rojas (2018) se ha ocupado de los factores que afectan la convivencia escolar: "sistema económico social”, menoscabo y respeto a los valores de convivencia, “mayor complejidad y heterogeneidad social”, "pérdida del liderazgo educativo", "mayor aparición y visibilidad de la violencia”.

\section{Conductas disruptivas}

En el estudio de Uruñuela Nájera (2007), se encuentran tres conductas disruptivas, agrupadas en la dimensión "centros educativos" (a su vez con dos dimensiones: centros de aprendizaje y centros de convivencia). En la dimensión centros de aprendizaje, se presentan tres tipos de conductas disruptivas: "falta de rendimiento, molestar en clase, y el absentismo". En la falta de rendimiento, se registran: pasividad, desinterés, apatía (no llevan el material de trabajo y no trabajan), permanencia fuera de clases, ubicación en sitio no autorizado, boicot (exámenes y otras actividades). El segundo tipo (molestar en clase) preocupa más a los profesores: "hablar de una manera reiterada", levantarse, 
moverse, mirar hacia atrás, cambiarse de sitio, "no dejar explicar al profesor", boicotear la clase, "impedir el desarrollo normal de la clase".

En la segunda dimensión, centro de convivencia, se presentan las conductas: "falta de respeto", "conflicto de poder" y "la violencia". La falta de respeto comprende: desobediencia y "contestar al profesor de forma impertinente y continuada"; por su parte, el "conflicto de poder" comprende: "desafío a la autoridad", "incumplimiento de sanciones" o "no aceptación de sus consecuencias". La violencia se disgrega en otras posibles conductas: "la violencia física, la verbal, la violencia psicológica, la social, la violencia contra las cosas y la violencia sexual y de género" (p. 104).

Rosário et al. (2012) han estudiado los comportamientos disruptivos: la distracción, la agresión, la trasgresión de las reglas. Las conductas disruptivas constituyen uno de los problemas más gravitantes en la escuela, cuyas conductas más frecuentes son: "falta de cooperación y mala educación, insolencia, desobediencia, provocación y agresión, hostilidad y abuso, impertinencia, amenazas, etc.” (Tattum, 1997, en Álvarez Hernández et al., 2016, p. 856).

De acuerdo con Quezada Campoverde (2018), los tipos de conductas disruptivas son: conductas de personalidad, conductas antisociales, conductas agresivas, conductas indisciplinadas, motrices, ruidosas, verbales, agresivas, de orientación en la clase.

\section{Consecuencias de la disrupción escolar}

Los estudios prueban que la disrupción afecta "más a los alumnos que a las alumnas"; por ejemplo, de cuatro afectados, tres son alumnos y una alumna (Uruñuela Nájera, 2007). Para Rosário et al. (2012), aunque los datos obtenidos en su estudio no certifican la asociación directa de los comportamientos disruptivos con el rendimiento académico en matemática, la literatura sí constata que los alumnos, quienes han 
experimentado un mejor rendimiento académico, tienen comportamientos positivos y adecuados en el aula durante la clase.

Los estudios realizados dejan la evidencia de que el fracaso escolar tiene una relación significativa con la disrupción. En este sentido, la disrupción es factor predictor del fracaso escolar, generando desligazón de las actividades escolares, la indiferencia de los estudiantes, el bajo rendimiento, la procrastinación de su comportamiento (Schunk y Ertmer, 2000; Rosario et al., 2009; 2010; Álvarez-García et al., 2011, en Rosário et al., 2012).

Por otra parte, los conflictos afectan las instituciones escolares, estas se constituyen en una especie de colección de conflictos. Los estudios de Capllonch Bujosa, Figueras Comas, and Lleixà Arribas (2014) suscriben varios conflictos encontrados en los centros escolares, entre los cuales se incluye la disrupción, "violencia psicológica, física y estructural, disrupción en las aulas, vandalismo, procesos de disciplina, bullying, acoso sexual, absentismo y deserción escolar, así como, problemas de seguridad” (p. 149). La disrupción escolar es una generadora de la presencia de otros conflictos en la institución educativa (Guerrero Machuca and Gutiérrez Acosta, 2018).

\section{Soluciones planteadas para corregir la disrupción}

La visión de conflicto-en la perspectiva de los alumnos, los docentes y los administradores_-ha cambiado durante los últimos años. "Se requiere de un análisis profundo, contextual y no estático de las 'personas y situaciones' generadoras de conflicto" (Orte-Socías and Ballester-Brage, 2007, p. 272). Es decir, se debe trabajar la prevención, porque estos problemas nacen en el seno de la convivencia; esta, con el conocimiento, el esfuerzo y el trabajo de todos, debe ser abordada preventivamente, evitando de esta manera la aparición de la "disrupción y disfunción, respuestas autoritarias, represoras o violentas, sentimientos de ofensa y humillación” (p. 272). 
A fin de plantear bien las soluciones, se debe interpretar muy bien las conductas disruptivas. "Todas tienen en común el rechazo al aprendizaje escolar y ponen de manifiesto el desajuste que existe entre los objetivos educativos del centro y los logros que obtiene el alumno; los objetivos educativos están muy lejos de la realidad del alumno, de su cultura experiencial y de sus intereses concretos” (Uruñuela Nájera, 2007, p. 104). Este investigador, además, advierte que las conductas disruptivas son señales y síntomas, marcan la distancia entre la enseñanza y el aprendizaje, dejando a la vista el enfrentamiento entre el profesor y el alumno. También destaca que es importante la comunicación, la empatía, desde el punto de vista educativo, entender objetivamente las conductas relacionadas con el centro educativo, con el centro de aprendizaje y con el centro de convivencia.

Otra alternativa para prevenir y desarrollar la resolución de conflictos en la escuela, es el trabajo de la educación física y deportiva en forma unida, "mediante una acción conjunta y coordinada entre familiares, grupo de iguales, colegio e instituciones deportivas” (Capllonch Bujosa et al., 2014, p. 154). La gestión del aula es muy importante para prevenir la conducta contraria a la convivencia escolar, con el propósito "de propiciar una mejora del clima de convivencia y gestión del aula" (Gil Espinosa, Chilón Garzón, and Delgado Noguera, 2016). También se recomienda, en la condición de solución, la formación de competencias sociodemográficas en los alumnos: pensamiento comprensivo ("compara, clasifica, analiza, sintetiza, averigua y se extraen conclusiones"), pensamiento crítico ("investigar fuentes, hacer interpretaciones, predecir efectos y razonar deductivamente") y pensamiento creativo: "produce ideas e imágenes, establece relaciones, crea metáforas y emprende metas" (Sanz de Acedo, 2010, en (Moncada Cerón and Gómez Villanueva, 2016). 
Por su parte, Rodríguez Biutrago (2016) plantea la gestión de las normas de comportamiento de la clases y la gestión del clima del aula; Erazo Santander (2016) sostiene que es indispensable realizar la identificación y la descripción de la intimidación escolar. Para Malagón Buitrago, Mateus Gómez, and Gómez Carrillo (2016), la convivencia escolar se constituye en el camino hacia la cultura de la paz. También aprecia y valora esta solución Nail Kroyer et al. (2018), quienes consideran que se experimentan tensiones y desafíos cuando se pretende elaborar las normas escolares para la resolución de problemas. Se propone trabajar la invocación disruptiva: "un camino para encontrar nuevas posibilidades y recursos, preguntas y relaciones", "la concepción, diseño y movilización inteligente de los espacios dinámicos de formación” (Pilonieta, 2017). García Barrera, Gómez Hernández, and Monge López (2017) resaltan la importancia de la atención a la diversidad, constituida en una propuesta metodológica.

Desde otro punto de vista, Domínguez Alonso et al. (2017) realizan una investigación con el propósito de prevenir y generar resoluciones de los conflictos escolares. En este sentido, se han plateados tres objetivos: "Identificar las principales publicaciones" sobre "la resolución de conflictos"; "analizar e interpretar la información bibliográfica"; "determinar las diferentes líneas de investigación, modelos de actuación y cuestiones polémicas en la prevención y resolución de conflictos” (p.152). Concluyen que se ejercerá la prevención y la solución de los conflictos escolares mediante el conocimiento de los aspectos siguientes: "el origen y la tipología de los conflictos, la orientación educativa y curricular, los estilos de enseñanza y prácticas didácticas, la formación del profesorado, los efectos y repercusiones del conflicto" (p. 154).

Asimismo, se propone realizar programas de convivencia para corregir las conductas disruptivas (Ortiz Flores, 2017; Miranda Tapia, 2018; Martinez Barrientos, 2018). En este caso, el arteterapia permite disminuir las conductas disruptivas de los 
estudiantes (Macas Bustamante, 2018). La gestión de la institución y del clima áulico permite la mejor convivencia escolar, generando compromiso, unidad y cooperación (Anchundia Chóez and Tuarez Triviño, 2018). Por ello, es importante tener en cuenta las pautas para el control de conductas disruptivas: autocontrol del adulto, aplicar el manual de convivencia, fomentar la reflexión en grupo, evitar situaciones negativas, ejercer de modelo, crear clases modelos (Quezada Campoverde, 2018).

En esta perspectiva, se requiere desarrollar algunas metodologías relacionadas con el aprendizaje y la convivencia escolar; es decir, estas metodologías ayudan para corregir las actitudes disruptivas de los alumnos (Ferriz Valero, García Martínez, and Arroyo Botella, 2018; Feria Cavero, 2018; Quedena Ruiz, 2018; Santamaría-Goicuria and Martinez Gorrochategi, 2018).

\section{Teorías y enfoques relacionados con la convivencia y la disrupción}

El presente estudio se construye sobre la base de algunas teorías y enfoques, cuyos alcances teóricos permiten argumentar las ideas y los comentarios prescritos. Por ejemplo, el enfoque sistémico nacido de la Teoría General de Sistemas; la Teorías de la Comunicación, la Teoría del Apego, el Modelo Ecológico, el Modelo Estructuralista de la Familia (Roiz, 1989; Espinal, Gimeno y González, 2006; en Armijos Piedra and Castro Ponce, 2018).

Según el enfoque sistémico, se afirma que el receptor recibe dos mensajes diferentes: uno relacionado con el acto comunicativo mismo y otro con el contenido de la comunicación, sin discriminar la meta comunicación (no entiende el código para entender al otro), generándose patologías comunicacionales y relacionales, en uno de los dos espacios: el espacio del grupo y el espacio de la familia (Bateson, 1959; Roiz, 1989; De Eusebio, 2015; en Armijos Piedra and Castro Ponce, 2018). 
De acuerdo con Bertalanffy (1976, en Armijos Piedra and Castro Ponce, 2018), la Teoría General de Sistemas es definida en los términos de un conjunto, cuyos elementos se interrelacionan en forma permanente generando reacciones diferentes, debido a que cada elemento constituye un subsistema con sus propias particularidades: grupos sociales, conglomerados sociales, familias, etnias, etc.

La familia es un sistema social, cuyas interacciones tienen un sentido, una ruta: de las simples hacia las complejas, en las cuales aparecen las incertidumbres y los fenómenos aislados (Morín y Pakman, 1994, en Armijos Piedra and Castro Ponce, 2018). La Teoría del Apego fue propuesta por Bowlby, para quien el desarrollo de los niños en sus dos dimensiones: emocional y cognitivo, obedece al apego seguro y estable con sus progenitores o con quienes los cuiden. Desde sus primeros años de vida, las interrelaciones con sus padres son valiosas, muy importantes y determinantes (Richaud de Minzi, Lemos y Mesurado, 2011; Moneta, 2014; en Armijos Piedra and Castro Ponce, 2018).

Para Watzlawick, Beavin y Jackson (1985, en Armijos Piedra and Castro Ponce, 2018), la comunicación es necesaria, también esencial, para las personas, sin la cual es imposible la interacción y la interrelación. La comunicación implica el proceso bidireccional; y en este contexto, la familia, sistema socializador del proceso comunicativo, tiene fronteras y límites internos, debido a sus subsistemas: conyugal, parental y filial. Los subsistemas se reorganizan, se relacionan y se modifican, en forma constantes, no son estáticos, son cambiantes (Minuchin, 2003; Varela y otros, 2015; Villarreal-Zegarra y Paz-Jesús, 2015; en Armijos Piedra and Castro Ponce, 2018).

De igual forma, el ambiente social (contexto social) es muy importante, pues permite estudiar y explicar la conducta y el desarrollo de los niños. El comportamiento de los niños es el resultado de su relación y de sus percepciones sobre las circunstancias 
y las características del ambiente social (Hernández y Ardón, 2015, en Armijos Piedra and Castro Ponce, 2018).

\section{Conclusiones}

A continuación, se registran las conclusiones relacionadas con los factores predictores, las consecuencias de la disrupción, sin dejar al margen las soluciones estudiadas en las investigaciones.

Los factores predictores de la disrupción son muchos; entre otros, las conductas o comportamientos de los profesores y de los padres de familia, los fenómenos migratorios, las condiciones sociales y económicas de la familia, la convivencia con la cultura y los valores, la pérdida del liderazgo educativo, dejando efectos visibles en los espacios y entornos del centro educativo.

En términos de efectos o consecuencias en los alumnos, la disrupción afecta más a los varones y menos a las mujeres; lo que deja a la vista de los involucrados el deficiente aprendizaje, el fracaso escolar, el bajo rendimiento, la indiferencia, la falta de ligazón de los estudiantes, la convivencia escolar inadecuada y negativa, y el clima áulico indebido e insoportable.

Por ello, a fin de prevenir y solucionar la disrupción, es necesario desarrollar análisis profundos y reflexivos sobre los contextos, los factores predictores y los efectos, en espacios donde intervengan los profesores, los alumnos, los padres de familia y los directivos. También es pertinente interpretar en forma acertada las conductas disruptivas, en un ambiente de comunicación empática entre los protagonistas, fomentando y valorando la convivencia; inclusive se recomienda desarrollar programas educativos y de convivencia, la gestión del aula y las normas de comportamiento, según la perspectiva de las teorías y los enfoques sistémicos. 


\section{Referencias}

Álvarez Hernández, M., Castro Pañeda, P., González-González de Mesa, C., Álvarez Martino, E., and Campo Mon, M. Á. (2016). Conductas disruptivas desde la óptica del docente: validación de una escala. Anales de Psicología, 32(3), 855-862. https://doi.org/10.6018/analesps.32.3.223251

Anchundia Chóez, L. A., and Tuarez Triviño, M. L. (2018). El clima áulico en la convivencia escolar campaña de concienciación y prevención de estupefacientes. Universidad de Guayaquil, Facultad de Filosofía, Letras y Ciencias de la Educación, Carrera Mercadotecnia y Publicidad. Guayaquil, Ecuador.

Armijos Piedra, T. R., and Castro Ponce, M. C. (2018). Estilos de crianza parental y las conductas disruptivas de los niños. Universidad Técnica de Machala, Unidad Académica de Ciencias Sociales, Carrera de Psicología Clínica. Machala, Ecuador.

Baños, R., Ortiz-Camacho, M. del M., Baena-Extremera, A., and Zamarripa, J. (2018). Efecto del género del docente en la importancia de la Educación Física, clima motivacional, comportamientos disruptivos, la intención de práctica futura y rendimiento académico Effect of teachers 'gender on the importance of physical education, motivati. Retos, (33), 252-257.

Beltrán, Y. I., Torrado, O. E., and Vargas, C. G. (2016). Prevalencia del hostigamiento escolar en las instituciones públicas de Bucaramanga-Colombia. Sophia, 12(2), 173-86. https://doi.org/10.18634/sophiaj.12v.2i.233

Buitrago Rojas, M. S. (2018). Factores que afectan la convivencia escolar en la Institución Educativa Técnica Agroindustrial Juan XXIII. Universidad del Tolima, Facultad de Ciencias de la Educación, Maestría en Educación. Ibagué, Tolima.

Capllonch Bujosa, M., Figueras Comas, S., and Lleixà Arribas, T. (2014). Prevención y resolución de conflictos en educación física: estado de la cuestión Prevention and conflict resolution in physical education: a review paper. RETOS. Nuevas Tendencias En Educación Física, Deporte Y Recreación, (25), 149-155.

Díaz Better, S. P., and Sime Poma, L. E. (2016). Convivencia escolar : una revisión de estudios de la educación básica en Latinoamérica. Revista Virtual Universidad Católica Del Norte, 49, 125-145.

Domínguez Alonso, J., Álvarez Roales, E., and Vázquez Varela, E. (2017).

Dimensiones predictivas del constructo violencia escolar en la educación secundaria obligatoria Predictors of violence in compulsory secondary education. Revista de Investigación Educativa, 35(2), 337-351. https://doi.org/10.6018/rie.35.2.259471

Erazo Santander, Ó. A. (2016). Identificación y descripción de la intimidación escolar en instituciones educativas del municipio de Popayán. Divers.: Perspect. Psicol, 12(1), 55-72. https://doi.org/10.15332/s1794-9998.2016.0001.04

Feria Cavero, L. E. (2018). Estrategias metodologicas de convivencia escolar. Pontificia Universidad Católica del Perú, Facultad de Educación.

Fernández-Larragueta, S., Fernández-Sierra, J., and Rodorigo, M. (2017). Expectativas socioeducativas de alumnas inmigrantes : escuchando sus voces Social and 
educational expectations of immigrant female students : listening to their voices. Revista de Investigación Educativa, 35(2), 483-498.

Ferriz Valero, A., García Martínez, S., and Arroyo Botella, J. . (2018). Metodología cooperativa para la mejora de actitudes disruptivas en educación física. Revista Internacional de Medicina Y Ciencias de La Actividad Física Y El Deporte, X(X), $1-17$.

García Barrera, A., Gómez Hernández, P., and Monge López, C. (2017). La atención a la diversidad en los moocs: una propuesta metodológica. Educación XX1, 20(2), 215-233. https://doi.org/10.5944/educXX1.13223

Gil Espinosa, F. J., Chilón Garzón, P., and Delgado Noguera, M. Á. (2016). Gestión de aula ante Conductas Contrarias a la Convivencia en Educación Secundaria Obligatoria Classroom management in problematic behaviors of coexistence in mandatory secondary education. Retos, (30), 48-53.

Guerrero Machuca, H. Y., and Gutiérrez Acosta, W. N. (2018). Manejo de la disrupción escolar generadora de conflicto en la institución educativa departamental agroindustrial Santiago de Chocontá e institución educativa municipal rural Río Frío de Zipaquirá. Universidad de la Sabana, Facultad de Educación, Maestría en Dirección y Gestión de Instituciones Educativas. Chía.

Ibarrola-García, S. (2014). El conocimiento práctico del profesor: ¿cuándo empieza todo? REDU Revista de Docencia Universitaria, 12(1), 219-238. Retrieved from http://search.ebscohost.com/login.aspx?direct=true \&db=a9h\&AN=96263680\&lan $\mathrm{g}=\mathrm{es} \&$ site $=$ ehost-live

Jacobo-Suárez, M. (2017). De regreso a "casa” y sin apostilla: estudiantes mexicoamericanos en México. Sinéctica, (48), 1-19.

Luciano, G., and Marín, L. (2011). Estudio de manifestaciones de violencia en las escuelas de la periferia de San Luis. Un estudio extensivo desde la mirada de los actores escolares. Fundamentos En Humanidades, XII(1), 93-109. Retrieved from http://search.ebscohost.com/login.aspx?direct=true $\& \mathrm{db}=\mathrm{a} 9 \mathrm{~h} \& \mathrm{AN}=89011053 \&$ lan $\mathrm{g}=\mathrm{es} \&$ site $=$ ehost-live

Macas Bustamante, Y. P. (2018). El arteterapia para disminuir las conductas disruptivas en estudiantes del noveno año paralelo " $b$ " de educación básica de la Unidad Educativa Marieta de Veintimilla, período 2017-2018. Universidad Nacional de Loja, Facultad de la Educación, el Arte y la Comunicación, Carrera de Psicología Educativa y Orientación. Loja, Ecuador.

Malagón Buitrago, E. L., Mateus Gómez, M. E., and Gómez Carrillo, S. E. (2016). La convivencia escolar, un camino hacia la cultura de la paz. Educación Y Calidad, (31), 43-58.

Martinez Barrientos, E. W. (2018). Programa "pitufeando" en la disminución de las conductas disruptivas y en mejora de las habilidades motrices básicas, 2017. Universidad César Vallejo, Escuela de Posgrado. Perú.

Martínez Ferrer, B., Musitu Ochoa, G., Amador Muñoz, L. V, and Monreal Gimeno, M. C. (2012). Estatus sociométrico y violencia escolar en adolescentes: implicaciones de la autoestima, la familia y la escuela. Revista Latinoamericana de Psicología, 44(2), 55-66. Retrieved from 
http://search.ebscohost.com/login.aspx?direct=true \&db=a9h\&AN=80215488\&lan $\mathrm{g}=\mathrm{es} \&$ site $=$ ehost-live

Miranda Tapia, M. (2018). Gestión de la convivencia escolar. Plan de acción. Pontificia Universidad Católica del Perú, Facultad de Educación.

Moncada Cerón, J. S., and Gómez Villanueva, B. (2016). Formación de competencias socioemocionales para la resolución de conflictos y la convivencia. Estudio de caso en la secundaria Sor Juana Inés de la Cruz Hidalgo, México. Revista Educación Y Desarrollo Social, 10(1), 112-133. Retrieved from https://dialnet.unirioja.es/descarga/articulo/5386127.pdf\%5Cnhttps://dialnet.unirioj a.es/servlet/extart?codigo $=5386127$

Nail Kroyer, O., Valdivia Guzmán, J., Gajardo Aguayo, J., Viejo Almanzor, C., Salas Salinas, R., and Romero Torres, G. (2018). Estudio de casos : tensiones y desafíos en la elaboración de la normativa escolar en Chile. Educ. Pesqui., São Paulo, 44, $1-22$.

Orte-Socías, C., and Ballester-Brage, L. (2007). Prevencion del comportamiento delictivo en la comunidad. Intervencion Psicosocial, 16(2), 269-281.

Ortiz Flores, C. E. (2017). Programa "Mejoremos la Convivencia” para corregir conductas disruptivas en estudiantes del segundo año, área de comunicación de la I.E. Santo Domingo de Guzmán del distrito de Moche, Trujillo, región de La Libertad, 2014. Universidad Nacional Pedro Ruiz Gallo, Facultad de Ciencias Históricos Sociales y Educación, Unidad de Posgrado, Maestría en Ciencias de la Educación. Lambayeque, Perú.

Penalva López, A. (2018). La convivencia escolar. Un reto del siglo XXI. Revista Electrónica de Investigación Y Docencia (REID), (20), 41-58. https://doi.org/10.17561/reid.n20.3

Picazo-Zappino, J. (2014). El suicidio infanto-juvenil: una revisión. Actas Españolas de Psiquiatría, 42(3), 125-132. Retrieved from http://dialnet.unirioja.es/servlet/extart?codigo $=4701495$

Pilonieta, G. (2017). Innovación disruptiva. Esperanza para la educación de futuro. Educación Y Calidad, (32), 53-64.

Quedena Ruiz, C. G. (2018). Disminución de las conductas disruptivas en el desarrollo de las sesiones de aprendizaje de los estudiantes del v ciclo de la I.E. "Carlos Augusto Salaverry” del distrito de Sullana. Pontificia Universidad Católica del Perú, Facultad de Educación.

Quezada Campoverde, D. E. (2018). Pautas de control de la disciplina para prevenir las conductas disruptivas dentro del aula de clase, dirigido a niños y niñas de sexto año de educación básica de la Escuela José Ingenieros, 2017-2018. Universidad Nacional de Loja, Facultad de la Educación, el Arte y la Comunicación, Carrera de Psicología Educativa y Orientación. Loja, Ecuador.

Rodríguez Biutrago, A. G. (2016). Gestión de normas de clase y clima de aula. Hojas $Y$ Hablas, (13), 57-71.

Rosário, P., Lourenço, A., Paiva, O., Rodrigues, A., Valle, A., and Tuero-Herrero, E. (2012). Predicción del rendimiento en matemáticas: efecto de variables personales, 
socioeducativas y del contexto escolar. Psicothema, 24(2), 289-295.

Santamaría-Goicuria, I., and Martinez Gorrochategi, A. (2018). El aprendizaje servicio, interculturalidad y justicia social: experiencias disruptivas y transformadoras con futuras maestras de educación infantil y primaria. Revista Qurriculum, (31), 97118.

Tapia Sisalema, J. O., and Rodríguez Baque, V. M. (2018). Inteligencia emocional en la convivencia escolar. Guía de actividades para la convivencia escolar. Universidad de Guayaquil, Facultad de Filosofía, Letras y Ciencias de la Educación, Carrera Educación Primaria, Modalidad Semipresencial. Guayaquil, Ecuador.

Uruñuela Nájera, P. M. (2007). Convivencia y disrupción en las aulas. Cuadernos de Pedagogía, (364), 102-107. Retrieved from https://0dialnet.unirioja.es.cataleg.uoc.edu/servlet/extart?codigo $=2190651$ 